\title{
Coherence imaging by use of a Newton rings sampling function
}

\author{
Adrian Gh. Podoleanu, George M. Dobre, David J. Webb, and David A. Jackson \\ Applied Optics Group, Physics Laboratory, University of Kent, Canterbury, CT2 7NR, UK
}

Received May 3, 1996

\begin{abstract}
We show that, with suitable optics in the arm of a Michelson interferometer, orthogonal galvo-scanning mirrors build a sampling function in the form of Newton rings when the two interferometer arms are matched. Using a low-coherence source, one can obtain transversal depth-resolved images. A fast display procedure using a storage oscilloscope was devised based on this method. (C) 1996 Optical Society of America
\end{abstract}

Different setups have been reported recently for optical surface profilometry by coherence interferometry. To build up a three-dimensional image of the target surface, an $(X, Y)$ scanning device and a $Z$-scanning device are used, where the $Z$ direction is approximately normal to the $(X, Y)$ surface. Usually, for each $(x, y)$ pixel in the frame, a $Z$ scan of the object is carried out, providing an interference signal only when the coherence plane defined by the reference arm is intersected by the object surface. ${ }^{1-4}$ The receiver detects these intersections from the amplitude of the Doppler beat signal whose frequency is determined by the speed of the mirror providing the $Z$ scan. From these data a transverse section at a given $z$ or an $(X, Z)$ or $(Y, Z)$ slice can be obtained. This longitudinal scan (LS) procedure ${ }^{1-3}$ has the advantage that the interference signal has a constant frequency, permitting good signal-to-noise processing. Reflected powers as low as $10^{-10}$ of the incident power have been detected in this way. ${ }^{3}$ The disadvantage is that a complete transversal image, i.e, a frame of pixels $(x, y)$ at a given $z$, is obtained only at the end of the process, after the scan in depth $(Z)$ has been completed for each $(x, y)$ pixel in the image. This issue is solved by the en face or the transversal scanning method in which a coherence $(x, y)$ frame can be obtained by modulation of the reference path by various devices such as electro-optic modulators and piezo components. ${ }^{4}$

Here we demonstrate a transversal scanning procedure with no modulation applied to the reference mirror. With suitable optics, scanning the light beam across the target with a galvanometric scanning mirror while the target arm is coherence matched with the reference arm leads to a modulation of the photodetected signal similar to that generated by a longitudinal scan.

The experimental arrangement is shown in Fig. 1. Light from a pigtailed superluminescent diode, SLD, enters a single-mode directional coupler, DC, where the output fibers form a Michelson interferometer. One arm (the reference) consists of microscope objective C1 and a mirror, $\mathrm{M}$, mounted upon a micrometer translation stage, MTS. The fiber lengths were cut to a 1-mm difference to keep the differential dispersion low, ${ }^{2}$ and the fiber ends were polished at $10^{\circ}$ to reduce the amount of returned light and consequently the level of shot noise. The other arm (the sensing arm) consists of microscope objective $\mathrm{C} 2$, the scanner block, SXY, lens L1, and the object under test
(OUT). Two triangle waveform generators, TX and TY, are used to drive the two mirrors, MX and MY, of the scanner block. The signal is collected by the photodetector, PD, amplified, and bandpass filtered in circuit BPF. A high-voltage amplifier is used to drive the $z$-modulation input of the analog storage oscilloscope, ASO.

Figure 1 shows the beam being deflected from point $\mathrm{O}$ on mirror MX when this mirror is tilted at different angles $\beta$. In this simplified approach the distance between mirrors MX and MY is neglected compared with the distance $x_{1}$ to lens L1; i.e., as regards the $Y$ scan, the beam can also be considered to originate from $\mathrm{O}$. The conjugate point of $\mathrm{O}$ is $\mathrm{O}^{\prime}$, and therefore the optical path lengths of all the reflected rays measured between mirror MY and $\mathrm{O}^{\prime}$ are equal. Let us consider a flat surface $\mathrm{S}$ as the object under test; it is oriented perpendicular to the system axis and intersected by the scanned beam at $\mathrm{N}$. The coherence surface $\Sigma$ defined by the length of the reference arm is given by the spherical surface of radius $r$ centered on $\mathrm{O}^{\prime}$. For each scanning angle $\beta$, two beams are superimposed upon the photodetector, one being reflected from point $\mathrm{N}$ and the second reflected by mirror $\mathrm{M}$; they can be equivalently considered to originate from point $\mathrm{P}$ on surface $\Sigma$. The optical path difference between the reference and the sensing arms is

$$
\Delta=2\left(\mathrm{O}^{\prime} \mathrm{N}-\mathrm{O}^{\prime} \mathrm{P}\right)=2\left(\frac{r}{\cos \alpha}-r\right) .
$$

Maxima are obtained when

$$
\Delta=2 M \lambda / 2,
$$

where $M$ is an integer. The angle $\delta \beta$ measured about point $\mathrm{O}$ between adjacent rays corresponding to two

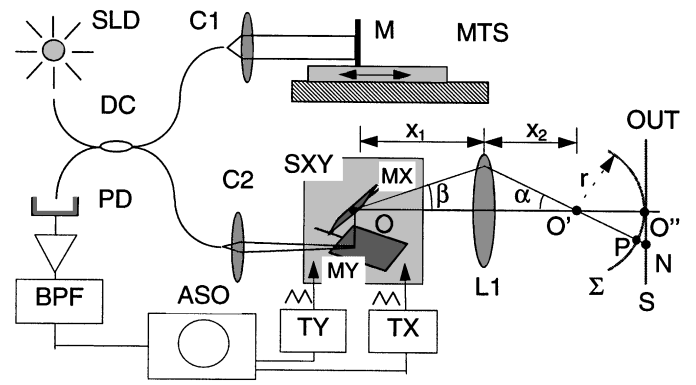

Fig. 1. Experimental arrangement. 
maxima is connected to the angle $\delta \alpha$ measured about point $\mathrm{O}^{\prime}$ and is given by

$$
\delta \beta=\frac{x_{2}}{x_{1}} \delta \alpha=\frac{x_{2}}{x_{1}} \frac{\lambda \cos ^{2} \alpha}{2 r \sin \alpha} \approx \frac{x_{2}}{x_{1}} \frac{\lambda}{2 r \alpha},
$$

where we have taken into account the distances $x_{1}$ and $x_{2}$ in Fig. 1 and assumed small angles in Eq. (3).

If mirror MX is driven by a triangular voltage signal of amplitude $U$ and frequency $F$ then

$$
\beta(t)=4 k U t F,
$$

where $k$ for our General Scanning G $120 \mathrm{~T}$ galvanometric scanning mirror pair is $4^{\circ} / \mathrm{V}=69.81 \mathrm{mrad} / \mathrm{V}$.

With Eqs. (1)-(4), the frequency at which maxima are encountered can be obtained as

$$
f=\frac{8 k U x_{1} F}{x_{2}} \frac{r \alpha}{\lambda} .
$$

One can obtain a similar expression by considering the speed of optical path difference variation in an equivalent LS setup in which the reference mirror is moved at a constant speed $v$. In this case the frequency of the Doppler beat signal amounts to $f=$ $2 v / \lambda$. Comparing this result with Eq. (5) leads to an equivalent speed:

$$
v=\frac{4 k U x_{1} F}{x_{2}} r \alpha .
$$

The geometric locus of the points on the surface $\mathrm{S}$ of maximum interference according to Eq. (2) is described by rings of radius $R_{M}=O^{\prime \prime} N$, given by

$$
R_{M}=\frac{2 r+M \lambda}{2}\left[1-\left(\frac{2 r}{2 r+M \lambda}\right)^{2}\right]^{1 / 2} .
$$

For small angles and low-order $M$, Eq. (7) becomes

$$
R_{M} \approx \sqrt{M r \lambda},
$$

which shows that the locus of the interference maxima is given by a relation similar to that describing Newton rings. However, our configuration differs from the Newton rings configuration presented in classical optics textbooks, which is based on a spherical element in contact with a planar element. ${ }^{5}$ Here the interfering rays producing Newton rings are coming from two different arms of the interferometer. When the reference-arm length is thought of as the equivalent of the distance to surface $\Sigma$ (as shown in Fig. 1), the interfering rays can be considered to coincide in directions $\left(P O^{\prime} O\right.$ and $\left.N O^{\prime} O\right)$. In addition, in this experiment there is no $\pi$ phase difference between the rays.

Given the coherence length $l_{c}$ of the source, the target area sampled by these Newton rings is

$$
A \approx(\pi / 2) l_{c} r .
$$

The analysis shows that we can consider the object to be sampled with a sampling function that in our case looks like concentric circles centered upon the system axis. In an $X-Y$ scan the beam maps the sampling function, with the returned signal being modulated in intensity at the frequency $f$ given by Eq. (5).

The scattering elements of a rough surface are displayed with this method when they subtend angles larger than $\delta \alpha$. The peak at frequency $f$ will be enlarged because of (a) the rough profile of the target, which acts as a random modulation factor, and (b) the extension $\Delta \alpha$ of the scatterer according to Eq. (5) (the higher $\Delta \alpha$ is, the higher the frequency spread $\Delta f$ ). It is therefore recommended that the regions where frequency $f$ is zero be avoided to reduce the relative spread $\Delta f / f$, i.e., the features to be scanned should be placed within regions where the Newton rings are closely spaced. A complete raster can be obtained with a long-persistence screen oscilloscope even when generators GX and GY are not synchronized.

We tacitly assumed in the theory that the diameter of the beam is much less than $\delta \alpha$. To ensure this and to improve the transversal resolution, we placed another lens, $\mathrm{L} 2$, of $2.5-\mathrm{cm}$ focal length at $\mathrm{O}^{\prime}$. The previously obtained relations are still valid when $r$ is replaced by $f$, the focal length of lens L2. The frequency of the signal driving the horizontal scanner was fixed at $F=20 \mathrm{~Hz}$ (signal generator GX), and the frequency of the signal applied to the vertical scanner (signal generator GY) was $0.25 \mathrm{~Hz}$. The amplitude of both signals was $0.25 \mathrm{~V}$. Using a plane mirror target, with the reference-arm coherence matched to the sensing arm and no bandpass filter after the photodetector, we adjusted the positions of collimator C2 and lenses L1 and L2 to bring the center of the displayed Newton rings to the center of the ASO, as shown in Fig. 2. This adjustment necessarily creates a situation in which the beam emerging from $\mathrm{C} 2$ is directed onto a point on the axis of rotation of each scanning mirror.

For a voltage amplitude of $0.4 \mathrm{~V}$ applied to the $X$ scanner and no voltage applied to the $Y$ scanner, the components of the photodetected signal are observed on a spectrum analyzer to span a spectrum up to $6 \mathrm{kHz}$. This compares well with the value of $5.4 \mathrm{kHz}$ obtained from Eq. (5) with $x_{1}=14.5 \mathrm{~cm}, x_{2}=10.2 \mathrm{~cm}$ and $\alpha=1.6^{\circ}$. The plane mirror was replaced with a 5 -pence coin whose feature of interest (an embossed

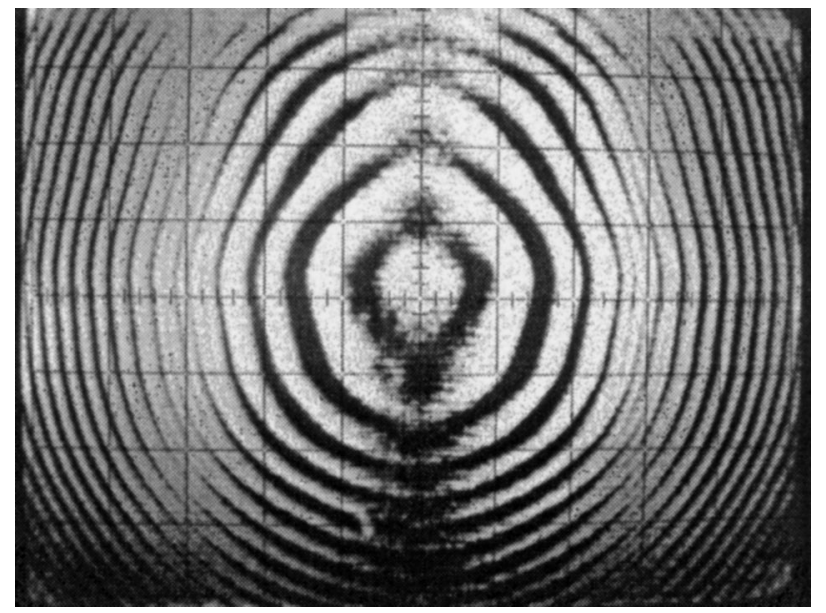

Fig. 2. Newton rings from a plane mirror; horizontal and vertical scales $50 \mathrm{mV} / \operatorname{div}(185 \mu \mathrm{m} / \mathrm{div})$. 

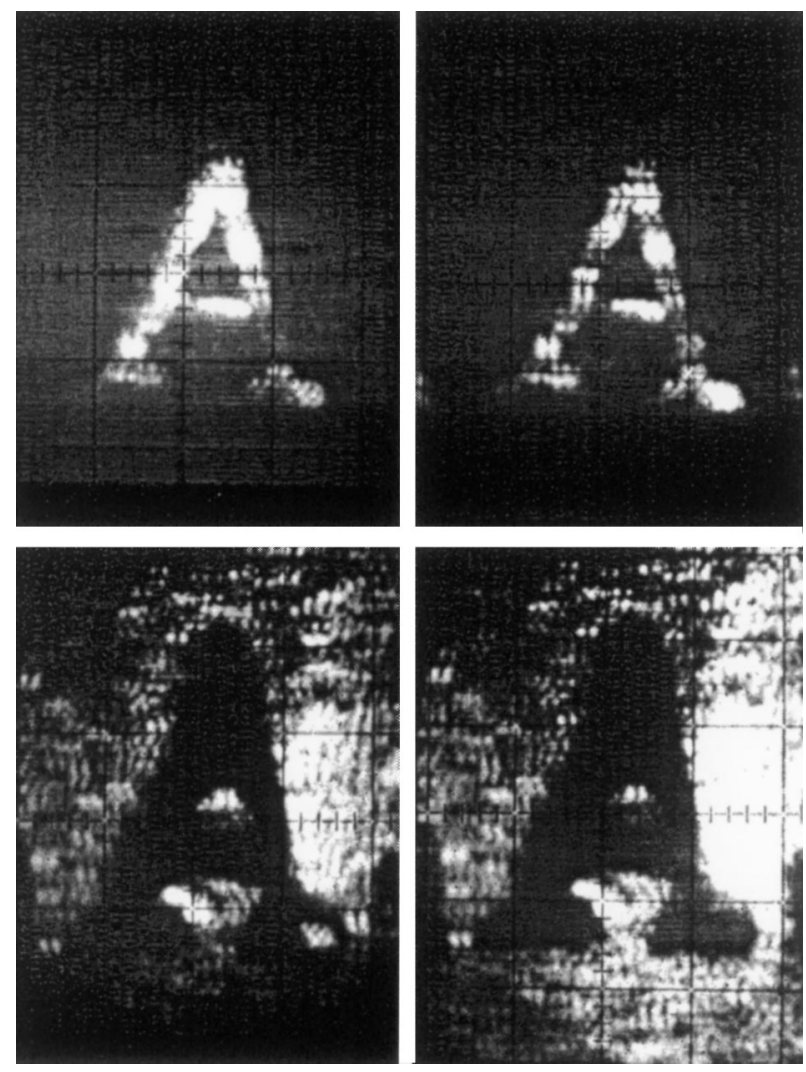

Fig. 3. Images obtained from a 5-pence coin. The reference path was increased in steps of $20 \mu \mathrm{m}$ between the top left the bottom right, reading across rows; horizontal and vertical scales $100 \mathrm{mV} / \operatorname{div}(370 \mu \mathrm{m} / \mathrm{div})$.

letter A) was placed at coherence. The bandpass filter was then adjusted to operate at $5 \mathrm{kHz}$ with a $Q$ factor of 5 ; thus only the upper part of the spectrum was selected. This resulted in a good rejection of the $1 / f$ noise and of the intensity modulation signal and also permitted the display of only those areas of the object that were sampled by denser Newton rings (where consequently the transversal resolution was better). A voltage amplitude of $0.4 \mathrm{~V}$ and a frequency of $0.25 \mathrm{~Hz}$ were also applied to the Y scanner. Four coherence images obtained at $20-\mu \mathrm{m}$ depth intervals are shown in Fig. 3, in which features of 75- $\mu \mathrm{m}$ width are clearly distinguishable. However, the minimum feature size on the object could be less than $40 \mu \mathrm{m}$ as determined from the average distance between the Newton rings in Fig. 2. The depth resolution given by the coherence length was measured as $35 \mu \mathrm{m}$ FWHM, in accordance with the value expected for a $20-\mathrm{nm}$ source linewidth and $\lambda=830 \mathrm{~nm}$.

A simple system to display micrometer-sized features in real time has been presented, with a frame being produced in $2 \mathrm{~s}$. Conventional devices and techniques are used, keeping the associated costs low. The system delivers an $(x, y)$ image for any fixed depth $z$. Wherever object features intersect the coherence surface a Doppler-like beat signal appears. We show and experimentally demonstrate that one can use this signal to obtain coherence images. Consequently, there is no vibrational noise induced by the movement of the translation stage, which is often found in LS setups. Furthermore, implementation is more straightforward than with other transversal scanning techniques.

The technique has the disadvantage that no modulation arises when the $\mathrm{S}$ surface coincides with the $\Sigma$ surface; i.e., a smooth surface with a curved profile matching the curvature of the rays emerging from $\mathrm{O}^{\prime}$ can be missed. However, this is a relatively unlikely case and can be excluded in practice when one is dealing with rough surfaces. Another disadvantage is connected with the frequency spread of the Doppler-like beat signal depending on the scanning angle, which may not permit bandwidth reduction below a certain value. Consequently, a lower signal-tonoise ratio than that in the LS approach results.

The main advantages of the method are its simplicity and quick display capability. Centering the Newton rings pattern with respect to the scan raster is essential to any en face technique. In these circumstances the method would permit a good adjustment of the setup before a more detailed investigation. Such an investigation could be a slow scan, multiple-slice, en face procedure with a large number of transversal pixels that uses the modulation of the reference arm.

In principle the method could be applied for faster scanning rates, if means such as increasing the input power are provided for maintaining a reasonably good value for the signal that is returned compared with the noise.

This Letter deals theoretically and experimentally with the particular case in which the scanner performs only rotation of the scanned beam. When the incident spot is moved away from the center (rotation axis) of the scanning mirror, the scanner itself would add a modulation of the path imbalance, as is usually the case with a polygon mirror. As the center of the Newton rings moves away from the center of the image, a reduced spread in the frequency spectrum and in an increase of the central frequency will result, which can create further advantages for imaging in terms of better transversal resolution and signal-to-noise ratio. We are currently investigating this procedure.

The authors acknowledge the support of the UK Engineering and Physical Sciences Research Council.

\section{References}

1. E. A. Swanson, J. A. Izatt, M. R. Hee, D. Huang, C. P. Lin, J. S. Schuman, C. A. Puliafito, and J. G. Fujimoto, Opt. Lett. 18, 1864 (1993).

2. B. Bouma, D. J. Tearney, S. A. Boppart, M. R. Hee, M. E. Brezinski, and J. G. Fujimoto, Opt. Lett. 20, 1486 (1995).

3. J. A. Izatt, M. R. Lee, D. Huang, J. G. Fujimoto, E. Swanson, C. P. Lin, J. S. Shuman, and C. A. Puliafito, Proc. SPIE 1877, 136 (1993).

4. M. R. Hee, J. A. Izatt, E. A. Swanson, and J. G. Fujimoto, Opt. Lett. 18, 1107 (1993).

5. A. F. Leung and J. E. Lee, Am. J. Phys. 59, 663 (1991). 